\title{
An improved prognostic model for stage T1a and T1b prostate cancer by assessments of cancer extent
}

\author{
Ramzi Rajab ${ }^{1}$, Gabrielle Fisher ${ }^{2}$, Michael W Kattan ${ }^{3}$, Christopher S Foster ${ }^{4}$, \\ Henrik Møller ${ }^{5}$, Tim Oliver ${ }^{1}$, Victor Reuter ${ }^{6}$, Peter T Scardino ${ }^{6}$, Jack Cuzick ${ }^{2}$ and \\ Daniel M Berney ${ }^{1}$, on behalf of the Transatlantic Prostate Group
}

${ }^{1}$ Centre for Molecular Oncology and Imaging, Barts and The London School of Medicine and Dentistry, Queen Mary University of London, London, UK; ${ }^{2}$ Cancer Research UK Department of Epidemiology, Mathematics and Statistics, Wolfson Institute of Preventive Medicine, Queen Mary University of London, London, UK; ${ }^{3}$ Department of Biostatistics and Epidemiology, Cleveland Clinic Foundation, Cleveland, OH, USA; ${ }^{4}$ Department of Cellular Pathology and Molecular Genetics, Liverpool University Hospital, Liverpool, UK; ${ }^{5}$ King's College London, Thames Cancer Registry, London, UK and ${ }^{6}$ Departments of Pathology and Urology, Memorial Sloan Kettering Cancer Center, NY, USA

\begin{abstract}
Treatment decisions on prostate cancer diagnosed by trans-urethral resection (TURP) of the prostate are difficult. The current TNM staging system for pT1 prostate cancer has not been re-evaluated for 25 years. Our objective was to optimise the predictive power of tumor extent measurements in TURP of the prostate specimens. A total of 914 patients diagnosed by TURP of the prostate between 1990 and 1996, managed conservatively were identified. The clinical end point was death from prostate cancer. Diagnostic serum prostate-specific antigen (PSA) and contemporary Gleason grading was available. Cancer extent was measured by the percentage of chips infiltrated by cancer. Death rates were compared by univariate and multivariate proportional hazards models, including baseline PSA and Gleason score. The percentage of positive chips was highly predictive of prostate cancer death when assessed as a continuous variable or as a grouped variable on the basis of and including the quintiles, quartiles, tertiles and median groups. In the univariate model, the most informative variable was a four group-split $(\leq 10 \%,>10-25 \%,>25-75 \%$ and $>75 \%)$; $(\mathrm{HR}=2.08,95 \%$ $\mathrm{Cl}=1.8-2.4, P<0.0001)$. The same was true in a multivariate model $\left(\Delta X^{2}(1\right.$ d.f. $\left.)=15.0, P=0.0001\right)$. The current cutoff used by TNM $(<=5 \%)$ was sub-optimal $\left(\Delta X^{2}(1\right.$ d.f. $\left.)=4.8, P=0.023\right)$. The current TNM staging results in substantial loss of information. Staging by a four-group subdivision would substantially improve prognostication in patients with early stage disease and also may help to refine management decisions in patients who would do well with conservative treatments.
\end{abstract}

Modern Pathology (2011) 24, 58-63; doi:10.1038/modpathol.2010.182; published online 10 September 2010

Keywords: conservative treatment; prostate cancer; stage; trans-urethral resection of prostate; watchful waiting

The advent of prostate-specific antigen (PSA) screening in the past 20 years has seen a decrease in the number of incidentally detected prostate cancers in patients treated by trans-urethral

Correspondence: Dr DM Berney, Centre for Molecular Oncology and Imaging, Barts and The London School of Medicine and Dentistry, Queen Mary University of London, Charterhouse Square, London EC1M 6BQ, UK.

E-mail: D.Berney@bartsandthelondon.nhs.uk

Received 13 May 2010; revised 20 July 2010; accepted 28 July 2010; published online 10 September 2010 resection (TURP) for benign prostate disease. The majority of neoplasms arise in the peripheral zones of the prostate making trans-rectal needle core biopsy the gold standard for confirming the diagnosis in patients with raised serum PSA. However, approximately $20 \%$ of prostate cancers arise in the transition zone ${ }^{1}$ and a minority of tumors may not cause a rise in PSA, especially those of low volume. Therefore, a minority of prostatic malignancies continue to be diagnosed on TURP specimens, especially in developing countries where PSA screening is not available. 
It has long been recognized that cancer extent in resection specimens may predict tumor behaviour. The current TNM pathological staging of prostate cancer on TURP chips stratifies patients into stage $\mathrm{T} 1 \mathrm{a}$ and T1b on the basis of the percentage area of tumor ( $<5$ vs $5 \%$ or greater) in the TURP specimen. ${ }^{2}$ This approach was based on a paper now nearly 30 years old and before the PSA era. ${ }^{3}$ Decisions on patient treatment are often made using this cutoff.

With the advent of tables or nomograms ${ }^{4}$ it is now recognized that the risk of progression can be given on a variable scale rather than the dichotomic TNM system, where valuable information may be lost and patients may receive inappropriate therapy. ${ }^{5}$ The Trans-Atlantic Prostate group was formed to study the natural history of prostate cancers with clinically localized cancers diagnosed by TURP or biopsy, and with diagnostic serum PSA and centrally assessed Gleason grading and tumor extent data available. We hypothesized that a more refined assessment of cancer extent in TURP specimens may aid treatment decisions in patients diagnosed by these means.

\section{Patients and methods}

The details of cohort assembly have been described previously. ${ }^{6}$ In brief, patients diagnosed with biopsy proven, localized prostate cancer between 1990 and 1996 were identified, by participating UK cancer registries. Exclusion criteria were age $>76$ years, no baseline PSA, invasive carcinoma within 5 years before diagnosis, or hormone treatment before biopsy. Patients were also excluded if they underwent radical prostatectomy, or radiotherapy within 6 months of diagnosis or had evidence of metastasis, a base line PSA $>100 \mathrm{ng} / \mathrm{ml}$ or died within 6 months of diagnosis. This was a practical method to ensure that initial treatment was not radical and patients had localized disease. The primary endpoint was defined as death from prostate cancer. Where available, death certificates were reviewed to verify cause of the death and survival outcomes were determined from medical records and cancer registry data. Last follow-up was December 2006.

Original diagnostic TURP biopsy specimens were reviewed to confirm the diagnosis and assign Gleason score. ${ }^{7}$ Tumor extent in TURP specimens was determined by calculating the fraction of TURP chips involved by tumor. The primary endpoint for this study was time to death from prostate cancer. Univariate and multivariate analysis were performed using a proportional hazard model. All $P$-values were two sided and $95 \%$ confidence intervals were based on a normal distribution.

\section{Results}

914 cases diagnosed by TURP were included in the cohort. The mean age at diagnosis was 69.4 years (range 49-76 years). The eligible cases were represented predominantly by patients receiving no initial treatment $(745,82 \%)$ with a minority receiving initial hormonal treatment $(169,18 \%)$. None of the patients received treatment (including $5 \alpha$ reductase inhibitors) before TURP. Median followup was 117 months (range 80-180 months).

The extent of cancer measured by the percentage of positive chips was available for 904 cases. In univariate analysis, this variable was highly predictive of prostate cancer death when assessed as a continuous variable or as a grouped variable on the basis of the quartiles (Figure 1), quintiles, tertiles (data not shown) or median groups (Table 1). The most informative expression was the variable in four groups $(\leq 10 \%,>10-25 \%,>25-75 \%$ and $>75 \%) ;\left(\mathrm{HR}=2.08,95 \% \mathrm{CI}=1.8-2.4, X^{2}(1\right.$ d.f. $)=151$ $P<0.0001)$. The variable based on two groups $\leq 10 \%$ and $>10 \%$, or the current TNM classification, $\leq 5 \%$ and $>5 \%$, (Figure 2) was also informative $\left(\mathrm{HR}=5.4,95 \% \mathrm{CI}=3.7-7.9, X^{2}(1\right.$ d.f. $)=107$

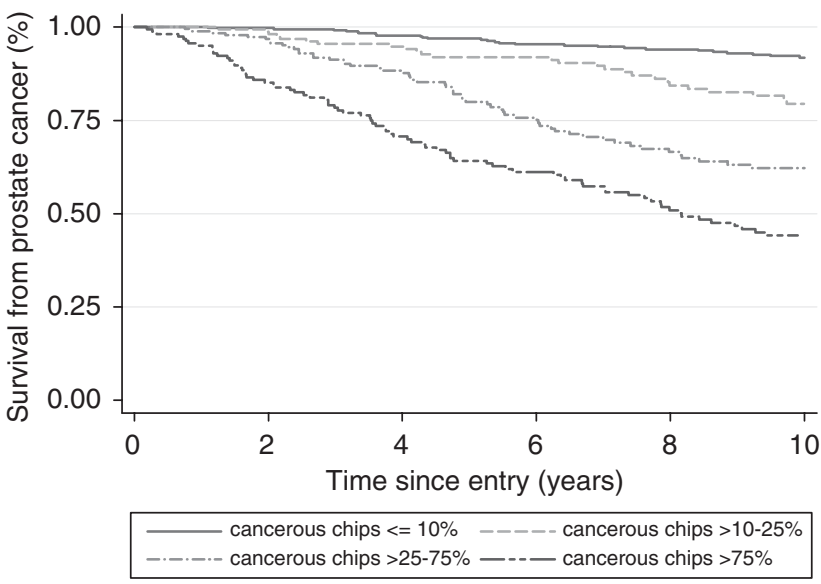

Figure 1 Prostate cancer cause specific survival as predicted by pathological assessment of extent of disease, percentage positive cancerous chips $(n=904)$.

Table 1 Univariate analysis of pathological assessments of extent of disease in predicting survival from prostate cancer in men with conservatively managed clinically localized disease $(n=904)$

\begin{tabular}{lccc}
\hline $\begin{array}{l}\text { \% Chips with } \\
\text { cancer }\end{array}$ & Cases & $\begin{array}{c}\text { Hazard ratio } \\
(95 \% \text { CI })\end{array}$ & $\begin{array}{c}\text { Prostate cancer } \\
\text { death at 10 years (\%) }\end{array}$ \\
\hline$\leq 5$ & 247 & $1^{\mathrm{a}}$ & 8 \\
$>5$ & 657 & $\begin{array}{c}\text { a.24 (2.7-6.7) } \\
\mathrm{X}^{2}(1 \text { d.f. })=57\end{array}$ & 32 \\
$\leq 10$ & 384 & $1^{\mathrm{a}}$ & 8 \\
$>10$ & 520 & $5.4(3.7-7.9)$ & 38 \\
& & $\mathrm{X}^{2}(1$ d.f. $)=107$ & \\
$\leq 10$ & 384 & $1^{\mathrm{a}}$ & 8 \\
$>10-25$ & 164 & $2.45(1.5-4.0)$ & 21 \\
$>25-75$ & 194 & $5.36(3.5-8.2)$ & 38 \\
$>75$ & 162 & $9.41(6.3-14.2)$ & 56 \\
& & $\mathrm{X}^{2}(1$ d.f. $)=151$ & \\
\end{tabular}

Abbreviation: CI, confidence interval.

${ }^{\mathrm{a}}$ Reference category. 


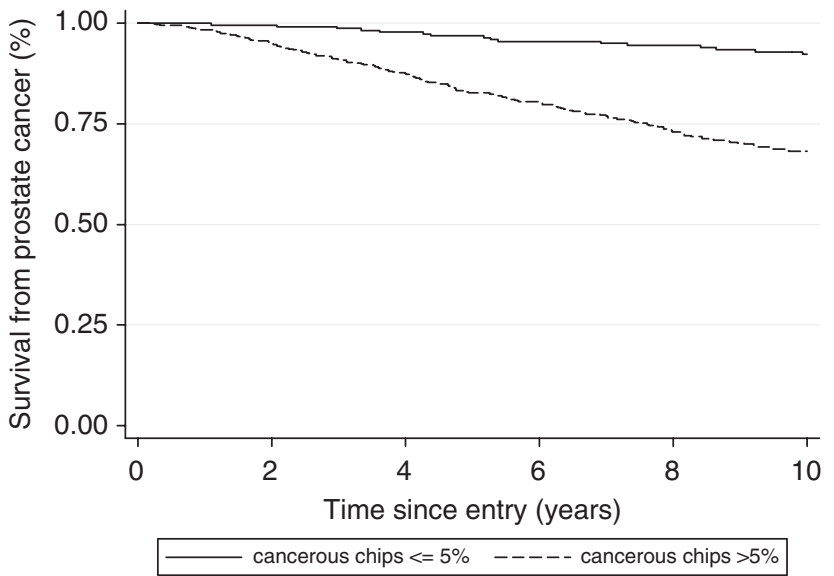

Table 2 The added predictive value of pathological assessments of extent of disease (TURP) for prostate cancer-specific survival, in a multivariate model, including Gleason Score (G) and baseline PSA (P) $(n=904)$

\begin{tabular}{|c|c|c|c|}
\hline Variable & $X^{2}$ & $\Delta X^{2}\left(\begin{array}{ll}1 & \text { d.f. })\end{array}\right.$ & P-value \\
\hline \multicolumn{4}{|l|}{ Basic model } \\
\hline $\begin{array}{l}\text { Gleason score }(\mathrm{G}) \\
(\leq 5,6,7,8, \geq 9)\end{array}$ & 200 & - & \\
\hline $\begin{array}{l}\mathrm{G}+\mathrm{PSA}(\mathrm{ng} / \mathrm{ml})(\mathrm{P})(4,>4-10 \\
>10-25,>25-50,>50-100)\end{array}$ & 228 & 28 & 0.0001 \\
\hline \multicolumn{4}{|c|}{ Basic model $(G+P)+$ added extent of disease variable (TURP) } \\
\hline TURP \% (continuous) & 241 & 13 & 0.0004 \\
\hline $\begin{array}{l}\text { TURP \% (median: } 0.4-15.7 \text {, } \\
15.75-100)\end{array}$ & 241 & 13 & 0.0002 \\
\hline $\begin{array}{l}\text { TURP \% (quartiles: 0.4-4.48, } \\
4.49-15.7,15.75-54,55-100 \text { ) }\end{array}$ & 239 & 11 & 0.0011 \\
\hline $\begin{array}{l}\text { TURP \% (groups: } \leq 10 \text {, } \\
>10-25,>25-75,>75)\end{array}$ & 243 & 15 & 0.0001 \\
\hline TURP \% (groups: $\leq 10,>10$ ) & 241 & 13 & 0.0004 \\
\hline TURP $\%(\leq 5,>5)$ & 233 & 5 & 0.023 \\
\hline
\end{tabular}

Abbreviation: PSA, prostate-specific antigen.

Table 3 Hazard ratios for multivariate model for prostate cancer death, based on Gleason Score, baseline serum PSA and pathological assessment of the extent of disease $(n=904)$

\begin{tabular}{lrc}
\hline Variable & Cases & Hazard ratio (95\% CI) \\
\hline $\begin{array}{l}\text { Gleason score } \\
\quad 55\end{array}$ & 54 & $0.8(0.2-2.6)$ \\
6 & 416 & $1^{\mathrm{a}}$ \\
7 & 222 & $1.71(1.1-2.7)$ \\
8 & 107 & $3.11(1.9-5.1)$ \\
9 or 10 & 105 & $5.86(3.6-9.6)$ \\
& & \\
PSA (ng/ml) & & $1^{\mathrm{a}}$ \\
$\leq 4$ & 327 & $1.5(0.9-2.5)$ \\
$>4-10$ & 191 & $2.29(1.4-3.7)$ \\
$>10-25$ & 175 & $2.19(1.3-3.6)$ \\
$>25-50$ & 128 & $2.62(1.5-4.4)$ \\
$>50-100$ & 83 & \\
TURP $(\%)$ & & $1^{\mathrm{a}}$ \\
$\quad \leq 10$ & 384 & $1.72(1-2.9)$ \\
$>10-25$ & 164 & $1.99(1.2-3.3)$ \\
$>25-75$ & 194 & $2.62(1.6-4.3)$ \\
$>75$ & 162 & \\
\hline
\end{tabular}

Figure 2 Prostate cancer cause specific survival as predicted by pathological assessment of extent of disease, percentage positive cancerous chips using current TNM classification (top) and a 10\% cutoff (below) $(n=904)$

$P<0.0001$ and $\mathrm{HR}=4.24, \quad 95 \% \quad \mathrm{CI}=2.7-6.7, \quad X^{2}$ (1 d.f.) $=57 P<0.0001$, respectively).

In multivariate analysis all forms of the variable extent of disease provided independent prognostic value over and above that of Gleason Score (grouped as $\leq 5,6,7,8$ and $\geq 9$ ) and baseline PSA (grouped as $\leq 4,>4-10,>10-25,>25-50$ and $>50-100 \mathrm{mg} / \mathrm{ml}$ ). In the multivariate model, the predictive value based on Gleason score $\left(X^{2}(1\right.$ d.f. $\left.)=200, P<0.0001\right)$ was increased by the addition of baseline PSA ( $X^{2}$ (1 d.f.) $=228, P<0.0001$ ) and increased further by the variable extent of disease. The added prognostic value of the different forms of the variable are shown in Table 2. The variable in four groups $(\leq 10 \%$, $>10-25 \%,>25-75 \%$ and $>75 \%$ ) added prognostic value ( $\Delta X^{2}$ (1 d.f.) 15.0), $P=0.0001$ ) that was higher than for any other extent of cancer variable (Table 2). The added value was almost as high for the simpler $10 \%$ cutoff variable ( $\Delta X^{2}(1$ d.f.) $13.0, P=0.0004)$ and lowest for the $5 \%$ cutoff variable corresponding to the current TNM classification $\left(\left(\Delta X^{2}(1\right.\right.$ d.f. $\left.)=5.0\right)$, $P=0.023)$. The full multivariate model, including Gleason score, baseline PSA and the four-group variable is shown in Table 3.

Abbreviations: CI, confidence interval; PSA, prostate-specific antigen. ${ }^{\mathrm{a}}$ Reference category.

In the group of patients restricted to those with no initial hormone treatment and with the percentage of positive chips data available $(n=738)$, in the multivariate model, results were similar to those for the unrestricted cohort, but the $5 \%$ cutoff was not significant $\left(\Delta X^{2}(1\right.$ d.f. $\left.)=2.6, P=0.1\right)$.

In Gleason subgroups, the value of extent of disease was also apparent when restricted to cases with Gleason score $<7(\mathrm{HR}=2.1,95 \% \mathrm{CI}=1.6-2.8$, $X^{2}(1$ d.f. $\left.)=23, P<0.0001\right)$ or Gleason score $\geq 7$ $\left(\mathrm{HR}=1.5,95 \% \mathrm{CI}=1.3-1.8, X^{2}(1\right.$ d.f. $)=28$ and the test for heterogeneity was not significant $(P=0.2))$. 
In addition, imunohistochemical staining results for the prognostic biomarker Ki-67 were available for 685 cases. Incorporating Ki-67, measured as percentage of positive cells, as a continuous variable, into the multivariate model, including Gleason score, baseline PSA and extent of disease, added further prognostic information for causespecific survival ( $\Delta X^{2}(1$ d.f.) $24.3, P<0.0001)$.

\section{Discussion}

Following the introduction of PSA testing in the early 1990s, there has been a large shift in the method of diagnosis of prostate cancer, towards ultrasound-guided biopsies of the peripheral zone and away from TURPs that sample the transition zone. Despite advances in accurately predicting the presence of prostate cancer before treatment for benign prostatic hyperplasia, ${ }^{8}$ a significant minority of cancers are still diagnosed by this method. In developing countries where ultrasound guidance is not available, the use of TURP is even higher.

Recent studies have explored the outcome of patients with incidentally diagnosed prostate cancer (T1a/b). Melchoir et al, found that reliable predictors of aggressive disease were lacking. ${ }^{9}$ Capitano et al showed that pre- and post-operative PSA and Gleason score following treatment for $\mathrm{BPH}$ were predictive of residual disease at radical prostatectomy. ${ }^{10}$ Another study, showed similar outcomes for biochemical recurrence and 10-year survival in patients with T1a/b compared with patients with T1c disease following radical prostatectomy, although patients with pT1b disease had a lower cancer-specific survival that was attributed to a higher frequency of adverse pathological factors in this group. ${ }^{11}$ Descazeaud et al, in a study of $144 \mathrm{~T} 1 \mathrm{a}$ prostate cancers with a 5-year follow-up developed a model using pre- and post-operative PSA, Gleason score, weight of resected tissue and clinical prostate weight to determine the risk of disease progression. ${ }^{12}$ They did not examine T1b cancers, and though a useful model for short term follow-up, no patient developed metastases and follow-up was by PSA recurrence alone. In the current study the end point was cause-specific survival. These findings indicate that patients with pT1 disease are a heterogeneous group with a proportion at higher risk of disease progression, in which better refinement is possible with standard parameters.

Articles examining tumor extent have concentrated largely on biopsy extent, including a recent evidence-based review of previous papers. ${ }^{13}$ TURP cancer volume is an issue that has not been addressed fully for over 20 years, and men diagnosed by this method may not have their risk of progression dealt with in a sophisticated manner.

Prostate staging has a complex history. In 1956 Whitmore developed the first staging system on clinical parameters. ${ }^{14}$ The death rate from stage 1 prostate cancer was low a non-aggressive approach to disease management was advocated. ${ }^{14}$ However, it later became apparent that a small proportion (approximately 10\%) of these patients would develop progressive disease, including distant metastases. ${ }^{15}$

Therefore, in 1959, Jewett subdivided Whitmore stage 1 patients into two groups by incorporating a histological assessment of the tumor went some way to addressing this issue by modifying the Whitmore staging system. ${ }^{16}$ In this system Stage A disease was defined as clinically unapparent tumor confined to the prostate gland without evidence of distant metastasis. Patients in this category were usually diagnosed following TURP for benign disease. These patients were then subdivided into two groups A1 and A2, by assessment primarily of the extent of tumor involvement in the TURP specimen (localized or diffused) and, second by the degree of differentiation (well differentiated or moderate and poorly differentiated).

In 1980 Cantrell et al, further refined the staging of clinically inapparent prostate cancer. ${ }^{3}$ They showed that the disease extent and histological grade in TURP specimens were two factors that most accurately predicted progression. However, there were only 117 patients in this study and there was progression in only 14 patients after 4 years. Despite these limitations it was found that subdividing disease extent into two groups, $5 \%$ or less and $>5 \%$ was a clinically useful determinant. Later studies similarly showed that disease extent was closely correlated with survival, clinical stage and risk of bone metastasis, with the advantage of less inter-observer variation than Gleason grading. ${ }^{17,18}$ This is the basis of the TNM sub-classification of localized $(\mathrm{T} 1 \mathrm{a} / \mathrm{b})$ prostate cancer, as its initial proposal by the UICC an ACJJ in 1992 to the current edition (sixth) in use today and the forthcoming seventh edition.

There are few studies considering progression after expectant treatment following TURP. ${ }^{19}$ Egevad et al examined the pathology on 305 men treated expectantly and found that both Gleason grade and extent of tumor provided prognostic information, but they used the $5 \%$ cutoff and did not present data on other means of measuring extent.

Methods of measuring cancer extent are variable in histopathology. The simplest method is the one used here, where the number of prostate chips involved by tumor is expressed as a percentage of the total number of chips. It has been argued that as the tumor usually does not occupy a whole chip, this provides an overestimate of cancer extent, and that an alternative approach used by some authors is to 'estimate' the amount of tissue involved, though in irregular chips containing irregular volumes of cancer. Estimating an irregular object area within an irregular object may be challenging. A computerbased or stereotactic approach is also possible, but we are unaware of it being used in routine practice. 
These issues were addressed by Foucar et al who compared the value of tumor extent measurements by a variety of methods in TURP specimens and correlated them with 5-year survival. ${ }^{20}$ They demonstrated that morphometric measurements of disease extent (absolute and percentage area of involved chips) were predictive of 5-year survival, but also that the percentage of chips involved by cancer closely matched the morphometric measurement in predictive value. The importance of the latter observation is that it makes time-consuming assessments of percentage area of disease extent unnecessary, without compromising the prognostic value of the assessment. Other groups have validated this method of counting prostatic chips. ${ }^{17,18}$

To our knowledge this data is the first to compare different subdivisions of tumor extent as Cantrell et al in 1981, and demonstrates that using a 5\% cutoff for tumor extent results in the loss of considerable information on the likelihood of tumor progression. Our study also confirms the findings of previous studies that show that assessments of the percentage of involved chips is predictive of survival in prostate cancer. ${ }^{3,21}$ It also confirms that the assessment of percentage chip involvement is a good way to assess tumor extent. We have demonstrated that the optimal assessment of extent would be by subdividing the percentage of involved chips into four groups $(\leq 10 \%,>10$ $\leq 25 \%,>25-\leq 75 \%$ and $>75)$ giving added prognostic value $(P=0.0001)$ over and above initial PSA and Gleason score in predicting prostate cancerrelated death. This information could be used to construct more refined nomograms for patients diagnosed on TURP.

The prognostic value of disease extent in biopsy specimens needs to be placed in context, with the role of novel prognostic biomarker markers. In this cohort Berney et al recently showed that the Ki-67 proliferation fraction of prostate cancer tissue in TURP specimens added prognostic information over and above age, serum PSA, cancer extent and Gleason sore. ${ }^{22}$ These novel biomarkers may further refine prognostic models.

Limiting factors include the retrospective nature of the cohort and selection criteria and variations in follow-up. Despite this heterogeneity of the study population in the follow-up period, these confounding factors are off-set by the cohort size, long followup, and that the availability of pre-operative PSA and contemporary Gleason scoring.

\section{Conclusion}

We have shown in the largest series published with PSA and contemporary Gleason scoring the utility and power of measuring the percentage of involved chips in TURP specimens to estimate the extent of disease and to predict long-term survival in prostate cancer patients.
We have demonstrated that the current T1a/b TNM subdivision could be greatly improved by adopting a four-group subdivision. This would enhance prognostication in patients diagnosed by TURP, allow the construction of better nomograms and may further refine management decisions in patients with low-to-intermediate risk disease who would qualify for active surveillance.

\section{Acknowledgement}

This work was supported by CRUK, the NIH and Orchid.

\section{Disclosure/conflict of interest}

The authors declare no conflict of interest.

\section{References}

1 Reissigl A, Pointner J, Strasser H, et al. Frequency and clinical significance of transition zone cancer in prostate cancer screening. The Prostate 1997;30:130-135.

2 Sobin L, Gospodarowicz M, Wittekind C. TNM Classification of Malignant Tumours. Wiley-Blackwell, 2009.

3 Cantrell BB, DeKlerk DP, Eggleston JC, et al. Pathological factors that influence prognosis in stage A prostatic cancer: the influence of extent versus grade. J Urol 1981;125:516-520.

4 Kattan MW, Zelefsky MJ, Kupelian PA, et al. Pretreatment nomogram that predicts 5-year probability of metastasis following three-dimensional conformal radiation therapy for localized prostate cancer. J Clin Oncol 2003;21:4568-4571.

5 Loeb S, Roehl KA, Helfand BT, et al. Complications of open radical retropubic prostatectomy in potential candidates for active monitoring. Urology 2008;72: 887-891.

6 Cuzick J, Fisher G, Kattan MW, et al. Long-term outcome among men with conservatively treated localised prostate cancer. $\mathrm{Br}$ J Cancer 2006;95: 1186-1194.

7 Epstein JI, Allsbrook Jr WC, Amin MB, et al. The 2005 International Society of Urological Pathology (ISUP) consensus conference on gleason grading of prostatic carcinoma. American J Surg Pathol 2005;29: 1228-1242.

8 Froehner M, Buck LM, Koch R, et al. Derivatives of prostate-specific antigen as predictors of incidental prostate cancer. Br J Urol 2009;104:25-28.

9 Melchior S, Hadaschik B, Thuroff S, et al. Outcome of radical prostatectomy for incidental carcinoma of the prostate. Br J Urol 2009;103:1478-1481.

10 Capitanio U, Scattoni V, Freschi M, et al. Radical prostatectomy for incidental (stage $\mathrm{T} 1 \mathrm{a}-\mathrm{T} 1 \mathrm{~b}$ ) prostate cancer: analysis of predictors for residual disease and biochemical recurrence. Eur Urol 2008;54:118-125.

11 Helfand BT, Mongiu AK, Kan D, et al. Outcomes of radical prostatectomy for patients with clinical stage T1a and T1b disease. Br J Urol 2009;104:304-309. 
12 Descazeaud A, Peyromaure M, Salin A, et al. Predictive factors for progression in patients with clinical stage T1a prostate cancer in the PSA era. Eur Urol 2008;53:355-361.

13 Harnden P, Shelley MD, Naylor B, et al. Does the extent of carcinoma in prostatic biopsies predict prostatespecific antigen recurrence? A systematic review. Eur Urol 2008;54:728-739.

14 Whitmore Jr WF. Hormone therapy in prostatic cancer. American J Med 1956;21:697-713.

15 Byar DP. Survival of patients with incidentally found microscopic cancer of the prostate: results of a clinical trial of conservative treatment. J Urol 1972;108: 908-913.

16 Jewett HJ. The present status of radical prostatectomy for stages A and B prostatic cancer. Urol Clin North Am 1975;2:105-124.

17 Humphrey R, Vollmer RT. The ratio of prostate chips with cancer: a new measure of tumour extent and its relationship to grade and prognosis. Hum Path 1988; 19:411-418.
18 Fan K, Peng CF. Predicting the probability of bone metastasis through histological grading of prostate carcinoma: a retrospective correlative analysis of 81 autopsy cases with antemortem transurethral resection specimen. J Urol 1983;130:708-711.

19 Egevad L, Granfors T, Karlberg L, et al. Prognostic value of the Gleason score in prostate cancer. Br J Urol 2002;89:538-542.

20 Foucar E, Haake G, Dalton L, et al. The area of cancer in transurethral resection specimens as a prognostic indicator in carcinoma of the prostate: a computer-assisted morphometric study. Hum Path 1990;21:586-592.

21 Epstein JI, Oesterling JE, Walsh PC. The volume and anatomical location of residual tumor in radical prostatectomy specimens removed for stage A1 prostate cancer. J Urol 1988;139:975-979.

22 Berney DM, Gopalan A, Kudahetti S, et al. Ki-67 and outcome in clinically localised prostate cancer: analysis of conservatively treated prostate cancer patients from the Trans-Atlantic Prostate Group study. Br J Cancer 2009;100:888-893. 